\title{
Personal protection with PBO-pyrethroid synergist-treated nets after 2 years of household use against pyrethroid-resistant Anopheles in Tanzania
}

Jackline L. Martin ${ }^{1,2^{*}}$, Franklin W. Mosha ${ }^{1}$, Eliud Lukole ${ }^{1}$, Mark Rowland ${ }^{3}$, Jim Todd ${ }^{2,3}$, Jacques D. Charlwood ${ }^{3}$, Jacklin F. Mosha ${ }^{2}$ and Natacha Protopopoff ${ }^{3}$

\begin{abstract}
Background: The spread of pyrethroid resistance in malaria vectors threatens the effectiveness of standard long-lasting insecticidal nets (LLIN). Synergist nets combine pyrethroid (Py) and piperonyl-butoxide (PBO) to enhance potency against resistance mediated by mono-oxygenase mechanisms. Our project assessed personal protection of the World Health Organization first-in-class PBO-Py LLIN (Olyset Plus) versus the standard LLIN (Olyset net) against pyrethroidresistant Anopheles gambiae sensu lato (s.l.) and An. funestus in North-West Tanzania after 20 months of household use.

Methods: From a household survey, 39 standard Olyset net and 39 Olyset Plus houses were selected. The physical integrity and hole index $(\mathrm{HI})$ of the nets were assessed, and resting mosquitoes were collected from inside nets and from room walls. The indoor abundance was estimated using CDC light traps and species identified using PCR. The bioefficacy of PBO and standard LLINs against wild Anopheles was assessed using 30-minute cylinder bioassays.
\end{abstract}

Results: Of 2397 Anopheles collected, 8.9\% $(n=213)$ were resting inside standard Olyset nets, while none were found inside Olyset Plus nets (PBO-Py LLINS) of any HI category. Resting density of blood-fed mosquitoes was higher on walls of sleeping rooms with Olyset nets compared to Olyset Plus (0.62 vs 0.10 , density ratio [DR]: $0.03,95 \% \mathrm{Cl} 0.01-0.13$, $p<0.001$ ). Mosquitoes were found inside Olyset nets of all WHO HI categories, but more were collected inside the more damaged nets $(H I \geq 643)$ than in less damaged (HI 0-64) nets (DR: 6.4, 95\% Cl 1.1-36.0, $p=0.037)$. In bioassay, mortality of An. gambiae s.l. was higher with Olyset Plus than with Olyset nets for new nets (76.8\% vs $27.5 \%)$ and nets used for 20 months (56.8\% vs 12.8\%); similar trends were observed with An. funestus.

Conclusion: The PBO-Py LLINs provided improved protection after 20 months of household use, as demonstrated by the higher bioassay mortality and absence of pyrethroid-resistant An. gambiae sensu stricto (s.s.) and An. funestus collected from inside Olyset Plus nets, irrespective of HI category, as compared to Olyset nets.

Keywords: Personal protection, An. gambiae, An. funestus, Insecticide resistance, Olyset plus, Olyset net, PBO, Piperonyl butoxide, Pyrethroid, Tanzania

*Correspondence: Iyimojaqueen@gmail.com

${ }^{1}$ Kilimanjaro Christian Medical University College, Moshi, United Republic of Tanzania

Full list of author information is available at the end of the article

\section{Background}

Long-lasting insecticidal nets (LLINs) are the cornerstone of malaria control in sub-Saharan Africa. The global malaria burden was reduced by $40 \%$ between 2000 and 2015, with insecticide-treated nets (ITNs) and LLINs making the largest contribution to control [1]. original author(s) and the source, provide a link to the Creative Commons licence, and indicate if changes were made. The images or other third party material in this article are included in the article's Creative Commons licence, unless indicated otherwise in a credit line to the material. If material is not included in the article's Creative Commons licence and your intended use is not permitted by statutory regulation or exceeds the permitted use, you will need to obtain permission directly from the copyright holder. To view a copy of this licence, visit http://creativecommons.org/licenses/by/4.0/. The Creative Commons Public Domain Dedication waiver (http://creativeco mmons.org/publicdomain/zero/1.0/) applies to the data made available in this article, unless otherwise stated in a credit line to the data. 
Since 2015, the annual malaria burden has not fallen any further in Africa [2]. The reasons for this are complex. While LLINs may provide personal protection for users even after becoming holed due to their insecticidal and excito-repellency effects [3], it has been shown that highly resistant phenotypes can penetrate the LLIN holes more readily to blood-feed and be found resting on inner surfaces [4, 5]. In such conditions, protective efficacy from malaria may be reduced when the physical condition of the net deteriorates [6]. Following the selection of high-level pyrethroid resistance arising from a combination of L1014S knock-down resistance $(k d r)$ mutation and mono-oxygenase metabolic mechanisms, also known as cytochrome P450s, in North-West Tanzania, even new intervention with standard LLINs may not reduce malaria transmission substantially [7-9].

In response to the rapid spread of pyrethroid resistance across Africa, the World Health Organization (WHO) has encouraged manufacturers to develop new types of LLINs that contain active ingredients with new modes of action to address the problem. Olyset Plus is a new-generation LLIN which incorporates pyrethroid permethrin and synergist piperonyl butoxide (PBO) to counter resistance to pyrethroids in mosquitoes caused by cytochrome P450-based metabolic mechanisms [10]. PBO inhibits the P450 enzymes which are responsible for detoxification of the pyrethroid before the neurotoxin reacts with its target site. In a cluster randomised trial (CRT) conducted in the same area as the present study, full coverage with PBO-Py LLINs (Olyset Plus) provided community protection against malaria for up to 2 years of use [11]. A further study, this one in Uganda, showed higher efficacy of PBO-Py LLINs compared to standard LLINs over the 18 months of the trial [12].

The aim of this study was to examine in an area of high pyrethroid resistance in North-West Tanzania the relationship between net holes and blood feeding of mosquitoes collected from inside pyrethroid-only and PBO-Py LLINs and from room surfaces containing these nets. The study provides evidence for differences in mosquito feeding success between Olyset Plus and standard Olyset nets under household conditions that serves as a proxy for personal protection from Olyset Plus after 20 months of use.

\section{Methods}

\section{Study area}

This study was embedded within a CRT whose main aim was to assess the community effect on malaria of a PBO-Py LLIN (Olyset Plus), in comparison with a standard pyrethroid LLIN (Olyset net), over 3 years of use [11]. The present study was conducted 20 months after the distribution of Olyset and Olyset Plus nets between
October 2016 and February 2017. The data collection was from four villages in Muleba district; Kyamyorwa and Ntungamo received Olyset Plus nets, and Kakoma and Kabirizi received Olyset nets. The four villages were selected because they had the highest density during the second post-intervention trapping year. Details of the study area have been reported previously [13]. In brief, the main malaria vectors in Muleba were An. gambiae s.s. (92\%), An. funestus s.l. (4\%) and An. arabiensis (4\%) [11]. Pyrethroid resistance frequency was over $90 \%$ in $A n$. gambiae s.l. and $45 \%$ in An. funestus s.l. exposed to the WHO diagnostic dose of permethrin (0.75\%). Addition of $\mathrm{PBO}$ in synergist bioassay resulted in restoration of susceptibility in $A n$. gambiae which indicates the involvement of cytochrome P450 in pyrethroid resistance [9].

\section{Household and net survey}

Ten to 12 houses were randomly selected from each of the four villages, and a maximum of two study nets in use were selected from each house at random until 40 nets of each type were reached. Information was collected from each house on household characteristics, LLIN ownership and usage. The selected LLINs were fitted over an Ifakara-style frame [14] and examined for presence, number, position and size of holes in accordance with the WHO LLIN guidelines [15]. Holes were categorised into four size classes (hole size $1=0.5-2 \mathrm{~cm}$ diameter; $2=2-10 \mathrm{~cm} ; 3=10-25 \mathrm{~cm}$ and $4=$ larger than $25 \mathrm{~cm}$ ) and weighted to estimate the hole area and hole index (HI) of each net [15]. HI was then allocated into three categories: "good" condition (HI 0-64), "acceptable" (65642) or "torn" ( $\geq 643)$.

\section{Mosquito collection}

Repeated mosquito collections were undertaken in each house during 6-10 visits over 5 months to obtain sufficient data for statistical analysis. Resting mosquito collection was conducted for 6 days in the villages with standard LLINs and up to 10 days in villages with PBOPy LLINs due to lower number of mosquitoes collected per day. Mosquitoes were collected from inside the selected study nets, and walls of rooms where the nets were installed were searched for mosquitoes using mouth and prokopack aspirators. An additional two nights of collection were done after completion of the resting collection visits using Centers for Disease Control and Prevention $(C D C)$ light traps installed at the foot of a bed fitted with the selected study net and run from $7 \mathrm{pm}$ to 7 am on each night. This was to gain further insight on overall mosquito densities and species entering the selected houses.

All mosquitoes collected were identified to species [16] and categorised to gonotrophic status microscopically 
(i.e. unfed, freshly fed, semi-gravid and gravid). An. gambiae s.l. were further identified to species using a realtime PCR Taq Man assay to distinguish An. gambiae s.s. from An. Arabiensis [17].

\section{Bioefficacy of the LLINs after 0 and 20 months}

The residual bioefficacy of the Olyset and Olyset Plus nets collected after 20 months of use was investigated on wild-caught An. funestus s.l. and An. gambiae s.l. using WHO cylinder test kits lined with the netting sampled from the study nets [18]. Netting was stapled to backing paper the same size as WHO test paper before insertion into the cylinder. Wild adult Anopheles of indeterminate age were collected using a prokopack and manual aspirator from inside houses (collection houses were different from the study houses selected for CDC light trapping and indoor resting collections) and were provided with glucose for 2-3 days to allow for blood digestion before testing. Anopheles were then exposed for 30 minutes to the net samples in the WHO cylinders, and mortality was recorded 24 hours later. Four to five replicates of 20 mosquitoes were exposed to each treatment. Bioefficacy tests were also carried out on unused, unwashed Olyset Plus and Olyset nets.

\section{Data analysis}

All data analysis was done using Stata version 13. Household characteristics, building materials, presence/absence of eaves, mosquito abundance, hole index and hole area were summarised according to net type and age.

Negative binomial regression was used to estimate the association between hole index and density of blood-fed Anopheles mosquitoes, adjusting for date and clustering by mosquitoes per net and repeated measures using robust standard errors.

\section{Results}

\section{Household and net characteristics}

The net surveys were carried out in 20 households with standard Olyset nets and 23 households with Olyset Plus nets. A total of 39 nets of each type were examined. Household and net characteristics were similar in the two groups. Average family size was 6.3 (standard deviation 1.8) in Olyset net households and 7 (standard deviation 2.6) in Olyset Plus households. In Olyset net villages, 15 (75.0\%, 95\% CI 51.1-89.6) of selected houses had mud walls and 11 (55\% 95\% CI 32.8-75.4) had open eaves, while in Olyset Plus villages, 22 (96.7\%, 95\% CI 73.299.4) had mud walls and 13 (56.5\%, 95\% CI 34.8-76.0) had open eaves. Over $80 \%$ of each net type had been in use for more than 20 months, and the median hole index and hole area at 20 months were similar between net types (Table 1).
Table 1 Insecticide-treated net characteristics and age in months when sampled

\begin{tabular}{|c|c|c|c|c|}
\hline & \multicolumn{2}{|l|}{ Olyset net } & \multicolumn{2}{|l|}{ Olyset Plus } \\
\hline & 0-5 months & $\begin{array}{l}20-21 \\
\text { months }\end{array}$ & 0-5 months & 20-21 months \\
\hline $\begin{array}{l}\text { Nets with } \\
\text { at least one } \\
\text { hole } \%,(n / N)\end{array}$ & $33 \%(2 / 6)$ & $100 \%(33 / 33)$ & $0 \%(0 / 4)$ & $94 \%(33 / 35)$ \\
\hline \multicolumn{5}{|l|}{ Hole area $\left(\mathrm{cm}^{2}\right)$} \\
\hline $\begin{array}{l}\text { Mean (std. } \\
\text { dev.) }\end{array}$ & $190(417)$ & 874 (1237) & - & 1526 (2689) \\
\hline Median (IQR) & - & $\begin{array}{l}573 \\
(65-1104)\end{array}$ & - & $640(95-1562)$ \\
\hline \multicolumn{5}{|l|}{ Hole index } \\
\hline $\begin{array}{l}\text { Mean (std. } \\
\text { dev.) }\end{array}$ & $155(340)$ & 712 (1008) & - & 1243 (2191) \\
\hline Median (IQR) & - & $467(53-898)$ & - & $521(77-1273)$ \\
\hline \multicolumn{5}{|c|}{ Hole index category \% (n) } \\
\hline $0-64$ & $67 \%(4)$ & $30 \%(10)$ & $100 \%(4)$ & $20 \%(7)$ \\
\hline $65-642$ & $17 \%(1)$ & $33 \%(11)$ & $0 \%(0)$ & $31 \%(11)$ \\
\hline$\geq 643$ & $17 \%(1)$ & $36 \%(12)$ & $0 \%(0)$ & $49 \%(17)$ \\
\hline
\end{tabular}

\section{Mosquito densities according to net type and collection method}

At 20 months post-intervention, when the study was carried out, a total of 2397 Anopheline mosquitoes were collected from standard Olyset nets and Olyset Plus nets and rooms using aspirators and light traps (Table 2). In CDC light traps, a higher density of mosquitoes were collected from households with standard Olyset nets (mean:17.5) than with Olyset Plus (mean: 6.4, DR: 0.37, 95\% CI 0.16-0.83, $p$ value $=0.015$ )

For standard Olyset nets, a mean of 0.9 mosquitoes were collected per net and three mosquitoes per room from wall collection. Overall, $13.1 \%(n=213)$ were collected from inside standard Olyset nets, $42.9 \%(n=699)$ from CDC light traps and $44.1 \%(n=719)$ from the room walls (Table 2). Among the 213 mosquitoes collected from inside the Olyset nets, $91 \%(n=193)$ were freshly blood-fed.

Zero mosquitoes were collected from inside Olyset Plus nets (Table 2). Mean number of blood-fed Anopheles resting on the walls was also much lower in rooms with Olyset Plus (mean: 0.10) compared to Olyset nets (mean: 0.63 , DR: $0.16,95 \%$ CI $0.04-0.69, p$ value 0.015 ). The two parameters are both indicative of high levels of personal protection.

The overall proportion of An. funestus collected relative to An. gambiae s.l. was much less in villages and houses with Olyset Plus $(5.9 \%, 45 / 766)$ than in villages and houses with standard Olyset net (43.6\%, 711/1631). 
Table 2 Indoor density of Anopheles mosquitoes according to collection method and net type

\begin{tabular}{|c|c|c|c|c|c|c|}
\hline & \multicolumn{3}{|l|}{ Olyset net } & \multicolumn{3}{|l|}{ Olyset Plus } \\
\hline & Light trap & On wall & Inside net & Light trap & On wall & Inside net \\
\hline Total number of collection events & 40 & 240 & 239 & 41 & 300 & 300 \\
\hline Mean no. Anophelines per collection $(N)$ & $17.5(699)$ & $3.0(719)$ & $0.9(213)$ & $6.4(264)$ & $1.7(502)$ & 0 \\
\hline Proportion An. gambiae s.I. (n) & $56.7(396)$ & $54.7(393)$ & $61.5(131)$ & $86.7(255)$ & $98.7(465)$ & 0 \\
\hline Proportion An. funestus ( $n$ ) & $43.3(303)$ & $45.3(326)$ & $38.5(82)$ & $13.3(39)$ & $1.3(6)$ & 0 \\
\hline Total An. gambiae s.l. tested for species & & 175 & 138 & & 126 & 0 \\
\hline Proportion An. arabiensis ( $n$ ) & & $1.1 \%(2)$ & $0.7 \%(1)$ & & $41.3 \%(52)$ & 0 \\
\hline
\end{tabular}

$N=$ total Anopheles collected

Among the An. gambiae s.l. resting on the wall identified to species, a much higher proportion of $A n$. arabiensis (41.3\%) relative to $A n$. gambiae s.s. were found in households with Olyset Plus than in households with standard Olyset nets (1.1\%).

\section{Blood-fed Anopheles mosquitoes collected while resting inside nets and on walls}

The average number of Anopheles mosquitoes found inside 20-month-old Olyset nets was higher in torn LLINs with $\mathrm{HI} \geq 643$ than inside standard Olyset nets in "good" condition (HI 0-64: DR: 6.38, 95\% CI 1.1136.0, $p$ value: 0.037 ) after adjusting for collection date (Table 3). No significant difference in density was found between nets in good and acceptable conditions. Most notably, no mosquitoes were found resting in Olyset Plus nets irrespective of net condition (Table 2). Density of blood-fed mosquitoes resting on room walls increased as hole index increased in rooms containing an Olyset net, while resting density remained stable across the three $\mathrm{HI}$ categories in Olyset Plus rooms (Table 3).

\section{PBO-pyrethroid LLIN bioefficacy monitoring}

Cylinder bioassays with wild, field-caught mosquitoes exposed to new Olyset nets showed mortality of $27.5 \%$ in An. gambiae s.l. and 27.2\% in An. funestus (Table 4). After 20 months of use, the bioassay mortality decreased to $12.8 \%$ in $A n$. gambiae s.l. and to $2.3 \%$ in An. funestus. The percentage mortality on exposure to new unwashed Olyset Plus nets was $76.8 \%$ in An. gambiae s.l. and $81.1 \%$ in $A n$. funestus, approximately three times greater compared to Olyset nets. After 20 months of use, the mortality on Olyset Plus decreased to $56.8 \%$ in An. gambiae s.l. and $25.3 \%$ in An. funestus, but mortality was still proportionately higher on Olyset Plus than on Olyset nets of similar age.

\section{Discussion}

The study showed that after 20 months of field use, the PBO-Py LLIN (Olyset Plus) continued to provide improved personal protection compared to the standard pyrethroid LLIN (Olyset net). There were no mosquitoes to be found resting inside Olyset Plus nets irrespective of LLIN condition or hole index, while inside Olyset nets, blood-fed Anopheles mosquitoes were found resting in

Table 3 Mean density of blood-fed Anopheles found inside Olyset net and resting on the walls of Olyset net and Olyset Plus rooms ( $\geq$ 20 months) by hole categories

\begin{tabular}{|c|c|c|c|c|c|c|c|c|c|}
\hline Hole index category & Total collection & $\begin{array}{l}\text { Mean } \\
\text { Anopheles } \\
\text { inside net }\end{array}$ & $\mathrm{DR}^{\mathrm{a}}$ & $95 \% \mathrm{Cl}$ & $p$ value & $\begin{array}{l}\text { Mean Anopheles } \\
\text { resting on wall }\end{array}$ & $D R^{a}$ & $95 \% \mathrm{Cl}$ & $p$ value \\
\hline \multicolumn{10}{|l|}{ Olyset net } \\
\hline $0-64$ & 60 & 0.5 & 1 & & & 0.05 & 1 & & \\
\hline $65-642$ & 66 & 0.2 & 0.43 & $0.06-3.30$ & 0.421 & 0.23 & 4.39 & $0.51-37.8$ & 0.178 \\
\hline$\geq 643$ & 72 & 2.0 & 6.38 & $1.11-36.0$ & 0.037 & 1.68 & 39.18 & $6.33-242.5$ & $<0.001$ \\
\hline \multicolumn{10}{|l|}{ Olyset Plus } \\
\hline $0-64$ & 54 & 0 & & & & 0.09 & 1 & & \\
\hline $65-642$ & 90 & 0 & & & & 0.10 & 1.14 & $0.37-3.47$ & 0.819 \\
\hline$\geq 643$ & 126 & 0 & & & & 0.12 & 1.97 & $0.56-6.91$ & 0.289 \\
\hline
\end{tabular}

a Density Ratio (DR) adjusted for collection date 
Table 4 Mortality of wild An. gambiae s.l. and An. funestus after 30-minute exposure and 24 hours holding with a new and a 20-month-old Olyset net and Olyset Plus net in WHO cylinder bioassays

\begin{tabular}{|c|c|c|c|c|}
\hline & \multicolumn{2}{|l|}{ Olyset net } & \multicolumn{2}{|l|}{ Olyset Plus } \\
\hline & 0 months & 20 months & 0 months & 20 months \\
\hline \multicolumn{5}{|l|}{ An.gambiae } \\
\hline Total tested & 80 & 78 & 82 & 81 \\
\hline Percent mortality at 24 hours (\%) & 27.5 & 12.8 & 76.8 & 56.8 \\
\hline $95 \% \mathrm{Cl}$ & $17.3-37.7$ & $10.4-15.3$ & $67.6-86.1$ & $46.9-66.6$ \\
\hline \multicolumn{5}{|l|}{ An. funestus } \\
\hline Total tested & 92 & 88 & 95 & 91 \\
\hline Percent mortality at 24 hours (\%) & 27.2 & 2.3 & 81.1 & 25.3 \\
\hline $95 \% \mathrm{Cl}$ & $22.5-31.8$ & $0-5.1$ & $65.2-96.9$ & $8.7-41.9$ \\
\hline
\end{tabular}

Zero mortality was observed after exposure to untreated nets in An. gambiae (tested $=79$ ) and An. funestus (tested =91)

nets of all physical conditions, increasing to a mean of two Anopheles per net in those of the highest hole index category.

According to WHO, a hole index that exceeds category HI 643 is considered unserviceable or non-protective in a standard pyrethroid LLIN [19]. The presence of bloodfed mosquitoes in a standard pyrethroid LLIN with a hole index less than this upper limit (i.e. across the range of HI 0-642 rather than just $\geq 643$ ) indicates that the categorisation of hole index adopted by WHO to distinguish between serviceable and non-serviceable categories has lost validity with respect to standard LLINs in environments that include a high proportion of highly resistant vectors. Similarly, in an area of high pyrethroid resistance in neighbouring Kenya, mosquitoes were collected inside standard LLINs in good and acceptable conditions, while none were found inside standard nets in an adjacent area comprised of susceptible mosquitoes [5].

The findings of the present study are also consistent with the observations made in the CRT, conducted in the same locality, in which no decrease in malaria prevalence occurred in the standard LLIN arm over the first 2 years of the trial, whereas in the PBO-Py LLIN arm, malaria prevalence decreased by almost one-half during the first 2 years [11]. The complete absence of mosquitoes in the Olyset Plus nets of any hole index category in the present household study after 20 months and low frequency of blood-fed mosquitoes on walls may be interpreted as restoration of protection by the PBO-Py LLIN despite the wide range of hole indices to be seen in these nets after almost 2 years of use. Pyrethroid and PBO content were not assessed for these nets; however, other study nets collected at the same time showed pyrethroid content of $16.7 \mathrm{~g} / \mathrm{kg}$ in Olyset and $12.2 \mathrm{~g} /$ $\mathrm{kg}$ in Olyset Plus nets, while PBO content was $1.6 \mathrm{~g} /$ $\mathrm{kg}$ compared to $9.2 \mathrm{~g} / \mathrm{kg}$ in new Olyset Plus nets [11], suggesting that $\mathrm{PBO}$ could still provide enhanced protection even at this much-reduced concentration. This restoration of protection against resistant mosquitoes seems analogous to the historic protection that was achieved when pyrethroid treatment of mosquito nets was first introduced 30 years ago to render untreated mosquito nets more protective against the pyrethroidsusceptible vector populations that were prevalent then [3]. With respect to PBO-Py LLINs, which are now being rolled out in preference to standard LLINs in many countries with pyrethroid-resistant vectors, the $\mathrm{HI}$ categorisation and absence of mosquitoes collected inside nets may still serve as a useful predictor of protection elsewhere in Africa until such time that resistance evolves against this class of net. When that point is reached, such highly resistant mosquitoes are predicted to survive and to be found resting inside PBO-Py LLINs.

CDC light trap surveillance was deployed to record the density of house-entering mosquitoes in Olyset net and Olyset Plus houses 20 months post-intervention. It was not our aim to have the study villages balanced in terms of mosquito density pre-intervention. Pre-intervention, the baseline density of Anopheles collected in CDC light traps in the two villages receiving Olyset Plus was 107.3 (median: 72) per night and 17.6 (median: 2) in the two Olyset net villages, which is an even greater difference than that seen 20 months after (Olyset Plus nets, mean 6.4; Olyset nets, mean 17.5) due to the cumulative effect of Olyset Plus on mosquito density post-intervention [11]. This re-emphasises the two effects of Olyset Plus compared to Olyset nets: greater An gambiae population control at the community level and enhanced personal protection for PBO-Py LLIN users.

Differences in blood feeding success between Olyset and Olyset Plus nets and, by inference, differences in 
personal protection have also been observed in experimental hut trials in pyrethroid-resistant areas elsewhere $[10,20]$. While no contemporary experimental hut trial of Olyset Plus nets versus standard Olyset nets has yet been reported in Tanzania against a resistant population of Anopheles gambiae, experimental hut trials conducted in the pyrethroid-resistant West African country of Burkina Faso showed a significantly reduced blood feeding rate in unwashed Olyset Plus nets compared to Olyset nets [20]. In Benin, blood feeding rate was higher in huts with Olyset nets washed 20 times, which is equivalent to at least 2 years and probably 3 years of use, compared to Olyset Plus nets according to WHO's proxy estimation of net longevity [10]. In the same experimental hut trial, it was also shown that the mortality of free-flying resistant mosquitoes induced by Olyset Plus (81\%) was approximately double that of Olyset nets $(42 \%)$, and this ratio did not change significantly after standardised washing over 20 cycles [10]. In a meta-analysis of experimental hut studies of several brands of PBO-Py LLINs, this new class of net was shown to induce 0.60 times less blood feeding than standard pyrethroid-only LLINs and to kill 1.85 times more pyrethroid-resistant mosquitoes [21]. In the context of the present Tanzanian study, major differences in mortality in residual bioassay were observed between Olyset Plus and standard Olyset nets ( 50\% difference for both An. gambiae s.l. and An. funestus) after 20 months of use. Of course, differences in mortality are more an indicator of toxicity and reduced mosquito longevity than of reduced blood feeding rate or personal protection. Nevertheless, the consistency in the trends in West African hut trials and Tanzanian household trials points towards higher efficacy of the PBO-Py LLINs compared to standard LLIN after 2 years of use.

The protection arising from the interaction between PBO and pyrethroid could be due to inhibition of metabolic resistance by $\mathrm{PBO}$, leading to restoration of enhanced knock-down and mortality. However, the excito-repellency of mosquitoes away from the net after contact with the PBO-pyrethroid surface may be more pertinent to personal protection than the classical synergism of pyrethroid resistance. For instance, LeClair et al. [13] observed that mosquitoes entering bedrooms were not killed after contact with Olyset Plus but showed increased excitability and a heightened escape reaction and capture rate in light traps present in the same room. More studies would be necessary to unravel the effects of PBO-Py LLINs on mosquito behaviour and synergism of metabolic resistance mechanisms.

The anthropophilic An. gambiae s.s. was the predominant vector species. A high proportion of An. arabiensis was collected from rooms with Olyset Plus nets compared to rooms with Olyset nets. An earlier CRT in this area reported limited involvement of An. arabiensis in malaria transmission based on its lower sporozoite rate and entomological inoculation rate (EIR) [22]. Over a decade ago, in response to the successful control of the primary vector An. gambiae s.s. in South-eastern Tanzania following increased coverage of standard LLINs in the universal coverage campaign [23], the ratio of $A n$. arabiensis to An. gambiae s.s. shifted towards higher $A n$. arabiensis. That species shift has been attributed in part to the more zoophilic An. arabiensis being less effectively controlled by standard LLINs than An. gambiae s.s. in sympatric populations of the species complex [24]. In the present study, the control of An. funestus and, to a lesser extent, of An. gambiae s.s. at both community and household levels by PBO-Py LLINs (and the failure to control these species in villages and households of the standard LLIN arm) may indicate the beginning of a species shift from An. funestus and An. gambiae s.s. to An. arabiensis in Olyset Plus net villages in NW Tanzania. However, no firm conclusion can be drawn due to the lack of data about species composition resting on the walls in these four villages at baseline.

For the comparison of residual activity (bioassay mortality) of Olyset Plus and Olyset nets, a WHO cylinder test with a 30-minute exposure was used [18]. The Olyset net performs poorly in the cone test even against susceptible mosquitoes (due to high excito-repellency of permethrin) with 3-minute exposure. In the more recent WHO 2013 guideline for evaluating LLINs [15], the tunnel test is proposed over the cone if cone mortality is low with a 3-minute exposure using susceptible mosquitoes. It is however difficult to test wild mosquitoes in tunnels and get them to host-seek or feed on guinea pigs. Cylinder tests were therefore preferred as bioassays on resistant mosquitoes but using a longer exposure than 3 minutes. Thirty minutes of exposure was used, as this is standard practice for indoor residual spraying (IRS) bioassays, and there is evidence for longer natural exposure time on nets in experiment huts when mosquitoes are resistant [25]. In the present study, even with 30-minute exposure, the cylinder test managed to achieve only $27.5 \%$ mortality against resistant $A n$. gambiae with a new Olyset and only $12.8 \%$ mortality on a 20 -month-old Olyset net. Mortality rates of An. gambiae s.l. and An. funestus in Olyset Plus nets declined in cylinder bioassays after 20 months of use. Despite this, the PBO-Py LLINs were still protective against mosquito feeding under field conditions.

\section{Conclusion}

The PBO-Py LLINs (Olyset Plus) provided improved protection after 20 months of household use, irrespective of hole index, as demonstrated by higher bioassay mortality 
and the absence of pyrethroid-resistant An. gambiae s.s. and An. funestus collected from inside Olyset Plus nets as compared to collections from inside standard LLINs after this period of use.

\author{
Acknowledgements \\ Special thanks to the community for allowing us to access to their houses and \\ nets, to the project technicians for collection, processing and identification of \\ mosquitoes, and to the molecular technicians at KCMUCo for the processing \\ and testing of mosquitoes.
}

\begin{abstract}
Authors' contributions
$J\llcorner M$ drafted the study protocol, implemented and supervised the field activities, performed molecular work and drafted the manuscript; FM contributed in the study design and supported the field activities; JT and JFW contributed in the analysis and sample size calculations; NP participated in the design of the study, implementation of activities, data analysis and revised the manuscript; EL \& JC participated in data collection, supervision and reviewed the manuscript; MR was the trial principal investigator, contributed to data interpretation and was a major contributor to writing and revising the manuscript. All authors read and approved the final manuscript.
\end{abstract}

\section{Funding}

This study was funded by the Joint Global Health Trials Scheme of the UK Department for International Development, Medical Research Council, and Wellcome Trust (MR/L004437/)

\section{Availability of data and materials}

The data sets generated and/or analysed during the current study are not public but are available from the corresponding author on reasonable request.

\section{Declarations}

Ethics approval and consent to participate

Written consent was obtained from household heads to participate in the study. Ethical approval for the main study was obtained from the Ethical Review Committee of the Tanzanian Medical Research Coordinating Committee (registration number NIMR/HQ/R.8a/Nol IX/1803) and the LSHTM. Further approval was obtained from the Ethical Committee of Kilimanjaro Christian Medical University College.

\section{Consent for publication}

Not applicable

\section{Competing interests}

The authors declare that they have no competing interests.

\section{Author details}

'Kilimanjaro Christian Medical University College, Moshi, United Republic of Tanzania. ${ }^{2}$ National Institute for Medical Research-Mwanza Centre, Mwanza, United Republic of Tanzania. ${ }^{3}$ London School of Hygiene and Tropical, London, United Kingdom.

Received: 10 August 2020 Accepted: 16 February 2021

Published online: 10 March 2021

\section{References}

1. Bhatt S, Weiss DJ, Cameron E, Bisanzio D, Mappin B, Dalrymple U, et al. The effect of malaria control on Plasmodium falciparum in Africa between 2000 and 2015. Nature. 2015:526(7572):207-11.

2. WHO: World Malaria Report. Geneva. Switzerland: World Health Organization; 2019. p. 2019.

3. Hill J, Lines J, Rowland M. Insecticide-treated nets. Adv Parasitol. 2006;61:77-128.

4. Asidi A, N'Guessan R, Akogbeto M, Curtis C, Rowland M. Loss of household protection from use of insecticide-treated nets against pyrethroidresistant mosquitoes, Benin. Emerg Infect Dis. 2012;18(7):1101-6.
5. Ochomo EO, Bayoh NM, Walker ED, Abongo BO, Ombok MO, Ouma C, et al. The efficacy of long-lasting nets with declining physical integrity may be compromised in areas with high levels of pyrethroid resistance. Malar J. 2013;12:368.

6. Rehman AM, Coleman M, Schwabe C, Baltazar G, Matias A, Gomes IR, et al. How much does malaria vector control quality matter: the epidemiological impact of holed nets and inadequate indoor residual spraying. PLOS ONE. 2011;6(4):e19205.

7. Protopopoff N, Matowo J, Malima R, Kavishe R, Kaaya R, Wright A, et al. High level of resistance in the mosquito Anopheles gambiae to pyrethroid insecticides and reduced susceptibility to bendiocarb in north-western Tanzania. Malar J. 2013;12:149.

8. West PA, Protopopoff N, Wright A, Kivaju Z, Tigererwa R, Mosha FW, et al. Indoor residual spraying in combination with insecticide-treated nets compared to insecticide-treated nets alone for protection against malaria: a cluster randomised trial in Tanzania. PLoS Med. 2014;11(4):e1001630.

9. Matowo J, Kitau J, Kaaya R, Kavishe R, Wright A, Kisinza W, et al. Trends in the selection of insecticide resistance in Anopheles gambiae s.l. mosquitoes in northwest Tanzania during a community randomized trial of longlasting insecticidal nets and indoor residual spraying. Med Vet Entomol. 2015;29(1):51-9.

10. Pennetier C, Bouraima A, Chandre F, Piameu M, Etang J, Rossignol M, et al. Efficacy of Olyset ${ }^{\circledR}$ Plus, a new long-lasting insecticidal net incorporating permethrin and piperonyl-butoxide against multi-resistant malaria vectors [corrected]. PLOS ONE. 2013;8(10):e75134.

11. Protopopoff N, Mosha JF, Lukole E, Charlwood JD, Wright A, Mwalimu $C D$, et al. Effectiveness of a long-lasting piperonyl butoxide-treated insecticidal net and indoor residual spray interventions, separately and together, against malaria transmitted by pyrethroid-resistant mosquitoes: a cluster, randomised controlled, two-by-two factorial design trial. Lancet. 2018;391(10130):1577-88

12. Staedke SG, Gonahasa S, Dorsey G, Kamya MR, Maiteki-Sebuguzi C, Lynd A, et al. Effect of long-lasting insecticidal nets with and without piperonyl butoxide on malaria indicators in Uganda (LLINEUP): a pragmatic, clusterrandomised trial embedded in a national LLIN distribution campaign. Lancet. 2020;395(10232):1292-303.

13. LeClair C, Cronery J, Kessy E, Tomas EVE, Kulwa Y, Mosha FW, et al. "Repel all biters": an enhanced collection of endophilic Anopheles gambiae and Anopheles arabiensis in CDC light-traps, from the Kagera Region of Tanzania, in the presence of a combination mosquito net impregnated with piperonyl butoxide and permethrin. Malar J. 2017;16(1):336

14. Lorenz LM, Overgaard HJ, Massue DJ, Mageni ZD, Bradley J, Moore JD, et al. Investigating mosquito net durability for malaria control in Tanzania - attrition, bioefficacy, chemistry, degradation and insecticide resistance (ABCDR): study protocol. BMC Public Health. 2014;14:1266.

15. WHO. Guidelines for laboratory and field-testing of long-lasting insecticidal nets. Geneva: World Health Organization; 2013.

16. Gillies MTC, Coetzee M. A supplement to the Anophelinae of Africa south of the Sahara (Afrotropical region). Publ S Afr Inst Med Res. 1987;55:1-43.

17. Bass C, Williamson MS, Field LM. Development of a multiplex real-time PCR assay for identification of members of the Anopheles gambiae species complex. Acta Trop. 2008;107(1):50-3.

18. WHO. Guidelines for testing mosquito adulticides for indoor residual spraying and treatment of mosquito nets. Edited by WHOPES. Geneva: World Health Organization; 2006.

19. WHO. WHO Guidance Note for Estimating the Longevity of Long-Lasting Insecticidal Nets in Malaria Control. Geneva: World Health Organization; 2013.

20. Toe KH, Muller P, Badolo A, Traore A, Sagnon N, Dabire RK, et al. Do bednets including piperonyl butoxide offer additional protection against populations of Anopheles gambiae s.l. that are highly resistant to pyrethroids? An experimental hut evaluation in Burkina Fasov. Med Vet Entomol. 2018:32(4):407-16.

21. Gleave K, Lissenden N, Richardson M, Choi L, Ranson H. Piperonyl butoxide (PBO) combined with pyrethroids in insecticide-treated nets to prevent malaria in Africa. Cochrane Database Syst Rev. 2018;11:CD012776.

22. Protopopoff N, Wright A, West PA, Tigererwa R, Mosha FW, Kisinza W, et al. Combination of insecticide treated nets and indoor residual spraying in northern Tanzania provides additional reduction in vector population 
density and malaria transmission rates compared to insecticide treated nets alone: a randomised control trial. PLoS ONE. 2015;10(11):e0142671.

23. Russell TL, Lwetoijera DW, Maliti D, Chipwaza B, Kihonda J, Charlwood JD, et al. Impact of promoting longer-lasting insecticide treatment of bed nets upon malaria transmission in a rural Tanzanian setting with preexisting high coverage of untreated nets. Malar J. 2010;9:187.

24. Kitau J, Oxborough RM, Tungu PK, Matowo J, Malima RC, Magesa SM, et al. Species shifts in the Anopheles gambiae complex: do LLINs successfully control Anopheles arabiensis? PLoS ONE. 2012;7(3):e31481.
25. Oxborough RM, N'Guessan R, Jones R, Kitau J, Ngufor C, Malone D, et al. The activity of the pyrrole insecticide chlorfenapyr in mosquito bioassay: towards a more rational testing and screening of non-neurotoxic insecticides for malaria vector control. Malar J. 2015;14:124.

\section{Publisher's Note}

Springer Nature remains neutral with regard to jurisdictional claims in published maps and institutional affiliations.
Ready to submit your research? Choose BMC and benefit from:

- fast, convenient online submission

- thorough peer review by experienced researchers in your field

- rapid publication on acceptance

- support for research data, including large and complex data types

- gold Open Access which fosters wider collaboration and increased citations

- maximum visibility for your research: over $100 \mathrm{M}$ website views per year

At BMC, research is always in progress.

Learn more biomedcentral.com/submissions 